\title{
Why People Put Each Other on Watches
}

With some regularity I saw the figure

Coming out of the blank fog.

In this unfathomable position of danger

I made my way forcefully past the crippled poodles

Named Gide, the old man in his nightly flee.

My favorite pastime is arriving home

Suitably in character,

One eye a little closed. 